\title{
Aircraft Pitch Control Design Using Observer-State Feedback
} Control

\author{
Hanum Arrosida*1, Mohammad Erik Echsony ${ }^{2}$ \\ 1,2 Politeknik Negeri Madiun \\ hanumarrosida@pnm.ac.id ${ }^{*}$, erik_sony@pnm.ac.id ${ }^{2}$
}

\begin{abstract}
Pitch is a movement of an aircraft achieved by tilting the elevator control which makes the nose of aircraft ascend or descend. This research will design a control system controlling the slope of the $x$-axis or pitch with the observer-state feedback to keep the aircraft always in the position setpoint, the stabilization of the pitch angle at the value of 0 radians. The applied controller was tested under three different conditions based on the variation of the gain value of $Q$ and $R$ affecting the observed gain matrix ( $L$ gain) and the matrix state-feedback gain (K gain). The variation with the gain value of $Q=10$ and $R=0.1$ was the best result of three kinds of testing performed with the fastest stabilization processing time, that was 7 seconds, to reach a steady state condition and the minimum pitch angle deviation value of 0.08 radians. The use of an observer has a significant influence on the pitch angle deviation of the aircraft. When using an observer, the pitch angle deviation value was 0.08 radians, and 0.2 radians without an observer. Larger result of pitch deviation angle will affect the stability of the aircraft's motion and cause the slope of the movement on the $x$-axis become greater so that the aircraft is prone to fall.
\end{abstract}

Keywords: Pitch control, Aircraft, Observer, State Feedback.

\section{Introduction}

In control theory, a state observer is a system providing an estimation of the presented internal state of the real system, starting from the measurement of input and output system in real-time [1]. It is usually applied in the simulation and becomes the basis for practical application purpose. The knowledge about state estimation is needed to solve problems related to control system [2]. For example, the problem of system stability by using state feedback. In most practical cases, the physical state cannot be determined by direct observation. On the contrary, the indirect effect from the internal state can be observed through output system [3].

Current aircraft is designed with an automatic control system to view and control some of the subsystems in the aircraft. The development of automatic control system has an important role in the area of aircraft movement control. To control the movement of aircraft there are 6 nonlinear equations [4].

Pitch is one of the aircraft movements to ascend or descend the aircraft's nose. Pitch can be controlled with the elevators on the tail of the aircraft. This research will design a control system controlling the slope of the $x$-axis or pitch with the observer-state feedback to keep the aircraft always in the position setpoint.

\section{Research Method}

The methods used in this research consists of several stages. The first stage of the implementation of this research is studying related literature and research. The second stage is designing the control method. The third stage is the development of system simulation and the application of the control method using MATLAB software. The last stage in this research is testing the overall system to collect and analyze data.

\subsection{Pitch Control Method}

A fixed-wing aircraft relies on its settled wing to lift up its body in order to fly. Usually, this wing is made from airfoil that has been designed in such ways to provide the needed lifting power, especially when an aircraft reaches a certain speed. A fixed wing type can float in the air because of its continuous movement with the power generated by the propeller. Figure 1 illustrates the model of an aircraft on pitch angle condition [5]. 


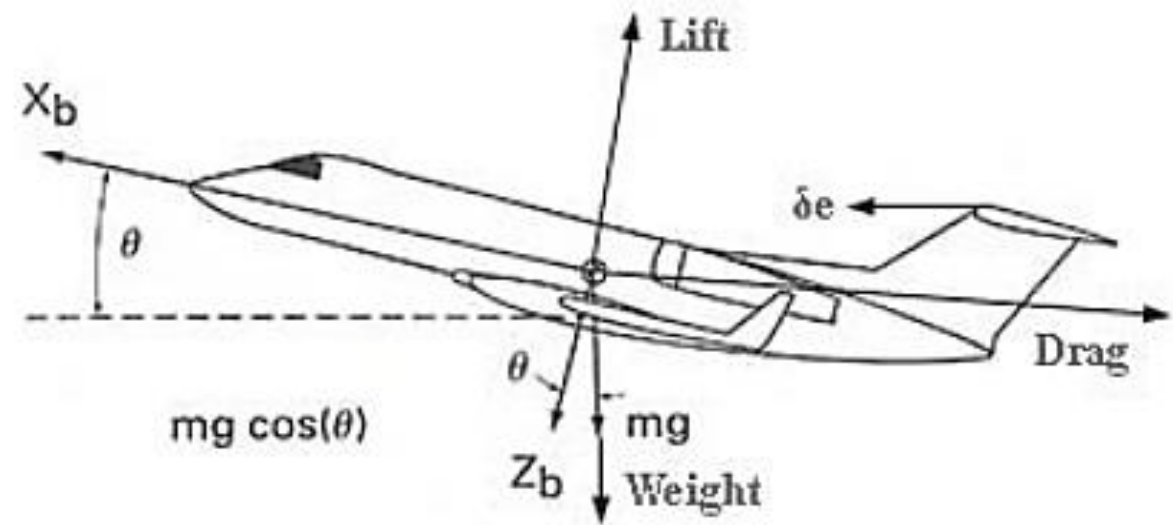

Figure 1. The Aircraft Flies at a Pitch Angle State

Based on the aircraft model used in Figure 1, several aircraft longitudinal equations are presented by Equation 1, 2, and 3. On this system, the input of the system was the angle of the slope on the elevator, while the output of the system was the pitch angle of the aircraft. All of those angles were measured in radian. Based on the data from Boeing Commercial Aircraft [6] and Flight Dynamic Principle [7], Equation 1, 2, and 3 can be expressed in the Equation 4, 5, 6 .

$$
\begin{gathered}
\dot{\alpha}=\mu \Omega \sigma\left[-\left(C_{L}-C_{D}\right) \alpha+\frac{1}{\left(\mu-C_{L}\right)} q-\left(C_{w} \sin \gamma\right) \theta+C_{L}\right. \\
\dot{q}=\frac{\mu \Omega}{2 i_{y y}}\left[\left[C_{M}-\eta\left(C_{L}+C_{D}\right)\right] \alpha+\left[C_{M}+\sigma C_{M}\left(1-\mu C_{L}\right)\right] q+\left(\eta C_{w} \sin \gamma\right) \delta\right] \\
\dot{\theta}=\Omega q \\
\dot{\alpha}=-2.02 \alpha+q+0.16 \delta \\
\dot{q}=-6.9868 \alpha-2.9476 q+11.7304 \delta \\
\dot{\theta}=q
\end{gathered}
$$

Afterwards, the function was converted to the domain of $S$ through $S$ transformation, resulted in Equation 7, 8, and 9. Utilizing few steps of algebra, Equation 10 stating the transfer function of the system was formulated. From Equation 10, an equation system in the state space was listed in the Equation 11 to 12 below.

$$
\begin{gathered}
s A(s)=-2.02 A(s)+Q(s)+0.16 \Delta(s) \\
s Q(s)=-6.9868 A(s)-2.9476 Q(s)+11.7304 \Delta(s) \\
s \Theta(s)=Q(s) \\
P(s)=\frac{\Theta(s)}{\Delta(s)}=\frac{11.7304 s+22.578}{s^{3}+4.9676 s^{2}+12.941 s}
\end{gathered}
$$




$$
\begin{gathered}
{\left[\begin{array}{c}
\Delta \dot{\alpha} \\
\Delta \dot{q} \\
\Delta \dot{\theta}
\end{array}\right]=\left[\begin{array}{ccc}
-2.02 & 1 & 0 \\
-6.9868 & -2.9476 & 0 \\
0 & 1 & 0
\end{array}\right]\left[\begin{array}{c}
\Delta \alpha \\
\Delta q \\
\Delta \theta
\end{array}\right]+\left[\begin{array}{c}
0.16 \\
11.7304 \\
0
\end{array}\right]\left[\Delta_{\delta e}\right]} \\
y=\left[\begin{array}{lll}
0 & 0 & 1
\end{array}\right]\left[\begin{array}{c}
\Delta \alpha \\
\Delta q \\
\Delta \theta
\end{array}\right]+[0]
\end{gathered}
$$

\subsection{Control Design}

The control design of the regulator system with the system state equation based on [8] was listed in the Equation 13, 14, and 15.

$$
\begin{gathered}
\dot{x}=A x+B u \\
y=C x \\
A=\left[\begin{array}{ccc}
-2.02 & 1 & 0 \\
-6.9868 & -2.9476 & 0 \\
0 & 1 & 0
\end{array}\right] \quad B=\left[\begin{array}{c}
0.16 \\
11.7304 \\
0
\end{array}\right], \quad C=\left[\begin{array}{lll}
0 & 0 & 1
\end{array}\right]
\end{gathered}
$$

To control the state-feedback, gain matrix $\mathrm{K}$ will be calculated.

$$
K=\left[\begin{array}{lll}
-0.2615 & 0.8363 & 1.0000
\end{array}\right]
$$

Using the state-feedback gain matrix $\mathrm{K}$, the control signal $u$ was given.

$$
u=-K x=-\left[\begin{array}{lll}
-0.2615 & 0.8363 & 1.0000
\end{array}\right]\left[\begin{array}{l}
x_{1} \\
x_{2} \\
x_{3}
\end{array}\right]
$$

The control signal $u$ was subsequently used to control the observer-state feedback.

$$
u=-K \tilde{x}=-\left[\begin{array}{lll}
-0.2615 & 0.8363 & 1.0000
\end{array}\right]\left[\begin{array}{l}
\tilde{x}_{1} \\
\tilde{x}_{2} \\
\tilde{x}_{3}
\end{array}\right]
$$

Based on [9], the Equation 16 was used to generate the observer gain matrix.

$$
\begin{gathered}
L=P C^{T} R^{-1} \\
L=\left[\begin{array}{ccc}
0.1759 & -0.1447 & -0.0040 \\
-0.1447 & 0.5096 & 0.1321 \\
-0.0040 & 0.1321 & 1.1243
\end{array}\right]\left[\begin{array}{l}
0 \\
0 \\
1
\end{array}\right][1]^{-1}=\left[\begin{array}{l}
-0.0040 \\
0.1321 \\
1.1243
\end{array}\right]
\end{gathered}
$$

Providing an estimate on $x(t)$ to be $\dot{\tilde{x}}$, the state observer was expressed in the dynamic system in Equation 17. 


$$
\begin{gathered}
\dot{\tilde{x}}=(A-L C) \tilde{x}+B u+L y \\
u=-K \tilde{x}
\end{gathered}
$$

By finishing equation 17, the obtained state observer value was,

$$
\begin{aligned}
& \dot{\tilde{x}}=\left\{\left[\begin{array}{ccc}
-2.02 & 1 & 0 \\
-6.9868 & -2.9476 & 0 \\
0 & 1 & 0
\end{array}\right]-\left[\begin{array}{c}
-0.0040 \\
0.1321 \\
1.1243
\end{array}\right]\left[\begin{array}{lll}
0 & 0 & 1
\end{array}\right]-\left[\begin{array}{c}
0.16 \\
11.7304 \\
0
\end{array}\right]\left[\begin{array}{lll}
-0.2615 & 0.8363 & 1.0000
\end{array}\right]\right\} \\
& {\left[\begin{array}{l}
\tilde{x}_{1} \\
\tilde{x}_{2} \\
\tilde{x}_{3}
\end{array}\right]+\left[\begin{array}{c}
-0.0040 \\
0.1321 \\
1.1243
\end{array}\right] y} \\
& =\left[\begin{array}{ccc}
-1.9782 & 0.8662 & -0.1560 \\
-3.9193 & -12.7578 & -11.8625 \\
0 & 1.0000 & -1.1243
\end{array}\right]\left[\begin{array}{l}
\tilde{x}_{1} \\
\tilde{x}_{2} \\
\tilde{x}_{3}
\end{array}\right]+\left[\begin{array}{l}
-0.0040 \\
0.1321 \\
1.1243
\end{array}\right] y
\end{aligned}
$$

By using Laplace transform in Equation 17 with an assumption that the initial condition was zero and to deal with $\tilde{X}$, Equation 19 can be obtained.

$$
\tilde{X}(s)=(s I-A+L C+B K)^{-1} L Y(s)
$$

The $\tilde{X}$ substitution in the Laplace transformation on Equation 18 was resulted in Equation 20.

$$
U(s)=-K(s I-A+L C+B K)^{-1} L
$$

Consecutively, the $U(s) / Y$ (s transfer function) can be expressed in the Equation 21 .

$$
\frac{U(s)}{Y(s)}=-K(s I-A+L C+B K)^{-1} L
$$

\subsection{System Simulation by Observer-State Feedback Control}

The designed system simulation and the application of control method were produced using MATLAB software. Figure 2 shows the block diagram of the system with a mathematical equation system in the form of the transfer function, while Figure 3 is a block diagram of the system with observer-state feedback control.

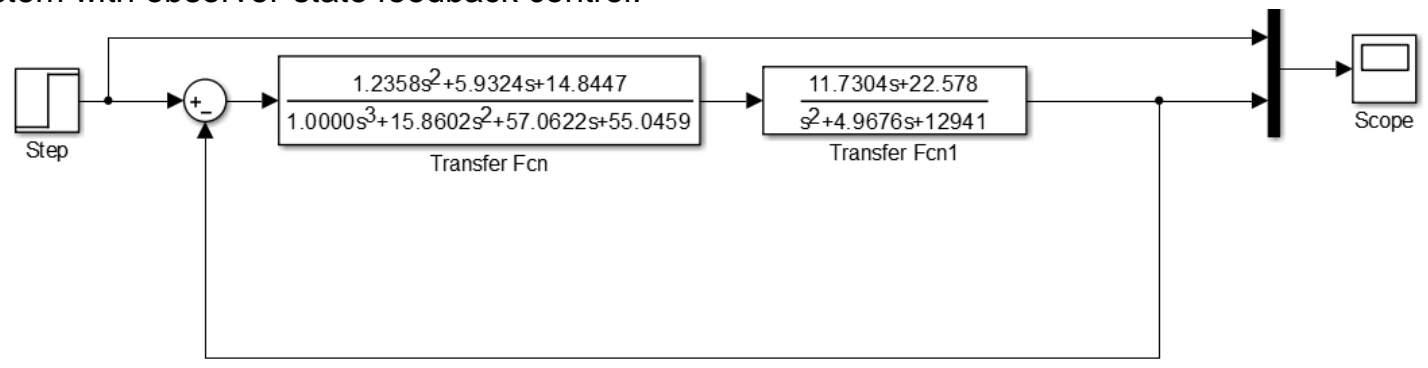

Figure 2. The Block Diagram of the System Using a Mathematical Equation System in The Form of The Transfer Function 
PLANT

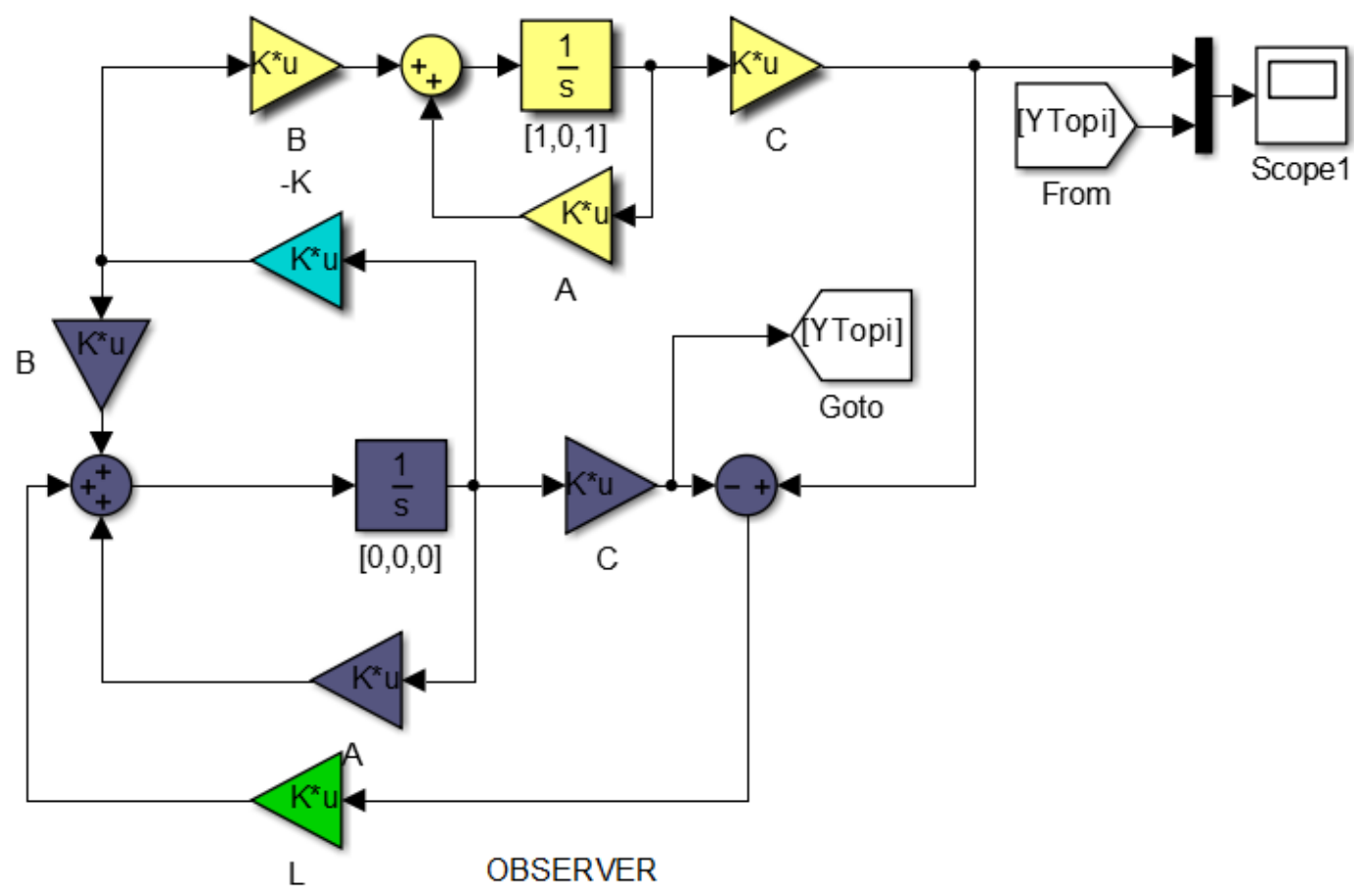

Figure 3. Block Diagram of The System with Observer-State Feedback Control

Figure 4 illustrates the data script in the MATLAB m-file for observer-state feedback control.

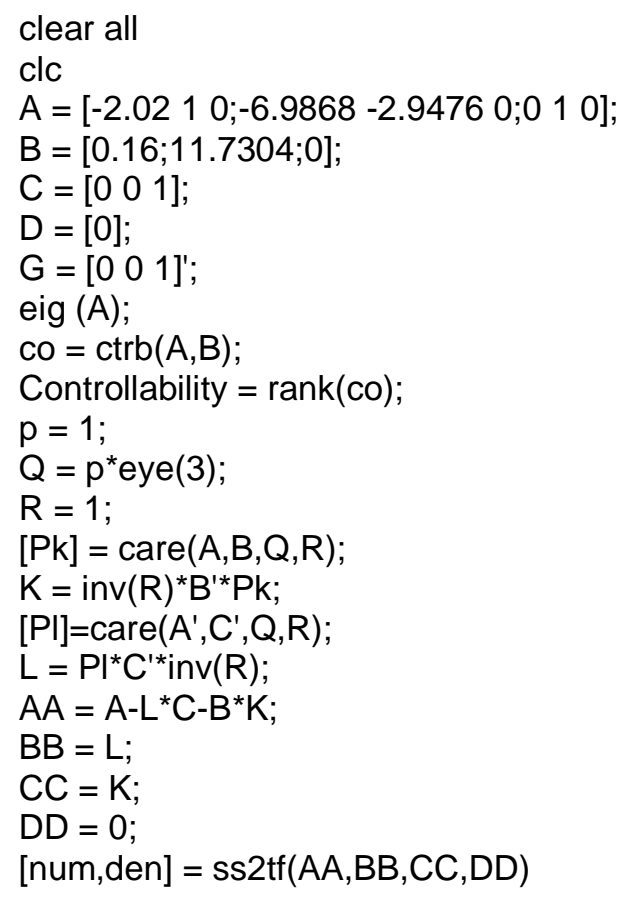

Figure 4. Data Script for Observer-State Feedback Control 


\section{Results and Discussion}

This research was tested with three variations, the first test evaluated the method of statefeedback observer control with the variation of $Q$ and $R$ on the same weight value, $Q=1$ and $R=1$. The second test utilizing a variation of the increased weight value of $Q$ and the decreased of $R$. The third test gave the variation decreased weight value of $Q$ and the increased of $R$. Three variation testing were used to determine the level of effectiveness from the method of statefeedback observer control. Variation was applied to determine the effect of changes in the value of the gain $Q$ and $R$ to change the value of parameter gain $\mathrm{K}$. Based on [10], the increasing value of the gain $Q$ caused the increasing value of the gain $K$. Meanwhile, the increasing value of the gain $R$ caused the deceasing value of the gain $K$.

\subsection{Testing Pitch Control using OSFC with $A$ Value of $Q=1$ and $R=1$}

Testing the pitch control was completed by the variation of $Q$ and $R$-value, as well as obersing the required time for the stabilization of pitch angle on the first test with the value of the weight $\mathrm{Q}$ and $\mathrm{R}$ based on to the Equation 22. The response of the testing results is shown in Figure 5 below.

$$
Q=\left[\begin{array}{lll}
1 & 0 & 0 \\
0 & 1 & 0 \\
0 & 0 & 1
\end{array}\right] ; R=1
$$

Based on the testing result using the value of the weight $Q=1$ and $R=1$, it was discovered that stabilizing pitch angle at 0-radian value with OSFC method required steady state time as much as 7.5 seconds and the magnitude of the pitch angle deviation of 0.08 radians. Moreover, the system response on the pitch angle without observer had a deviation of 0.2 radians and required steady state time as much as 7.5 seconds for stabilizing the pitch angle at 0 radians. Thus, it can be stated that the use of OSFC method was more effective because the stabilization process had smaller deviation than without the presence of the observer.

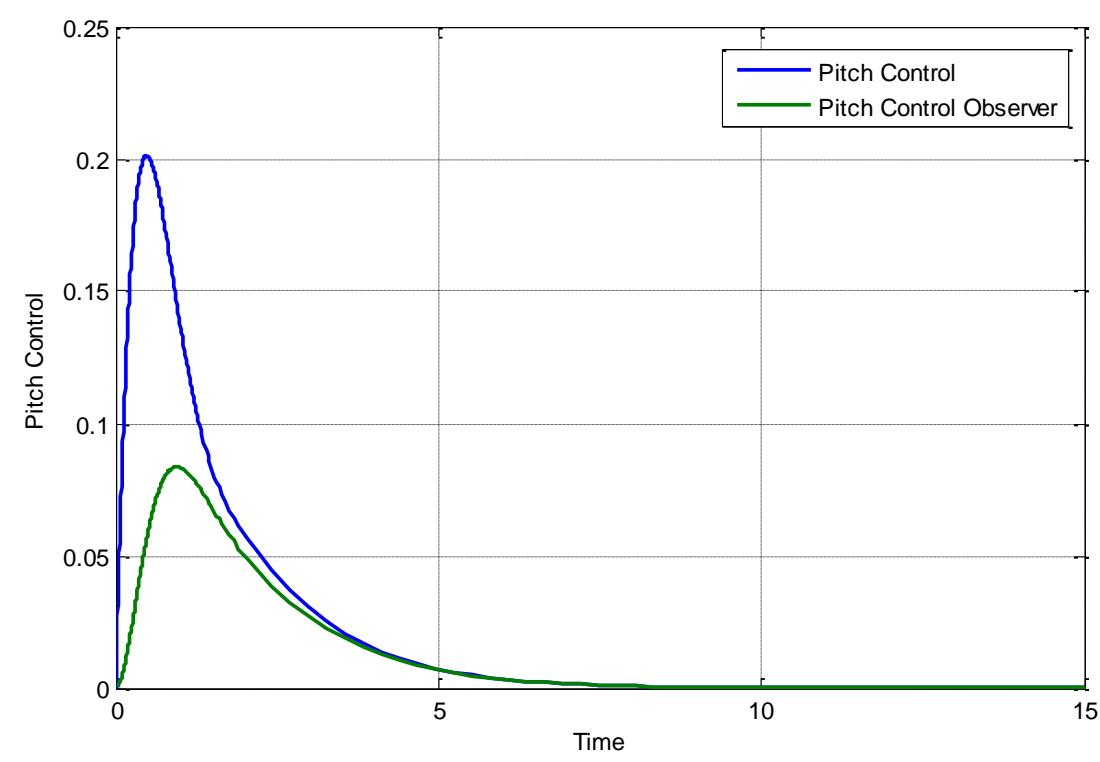

Figure 5. Testing Responses of Control Pitch with OFSC Weights $Q=1$ and $R=1$

\subsection{Pitch Control Test using OSFC with Value of $Q=10$ and $R=0.1$}

The second test was performed using $Q=10$ and $R=0.1$. Equation 23 states the matrix used for $Q$ and $R$ in the second test. Using the same method with the first test, the data of the relationship between the magnitude of the pitch angle versus the time were obtained. The responses of the second test results are depicted in Figure 6 below. 


$$
Q=\left[\begin{array}{ccc}
10 & 0 & 0 \\
0 & 10 & 0 \\
0 & 0 & 10
\end{array}\right] ; R=0.1
$$

Based on the chart of the second test result listed above, it is known that the use of the OSFC controller on aircraft pitch control has pitch angle deviation as much as 0.08 radians for the stabilization of the angle at 0 radians. On the other hand, the pitch control without observer shows a deviation angle of 0.2 radians. The duration required for the application of both methods in reaching the steady state is 7 seconds. From these results, the application of pitch control either with the presence of the observer or not requires identical duration in the stabilization process at 0 radians. The difference lies on the magnitude of the pitch angle deviation.

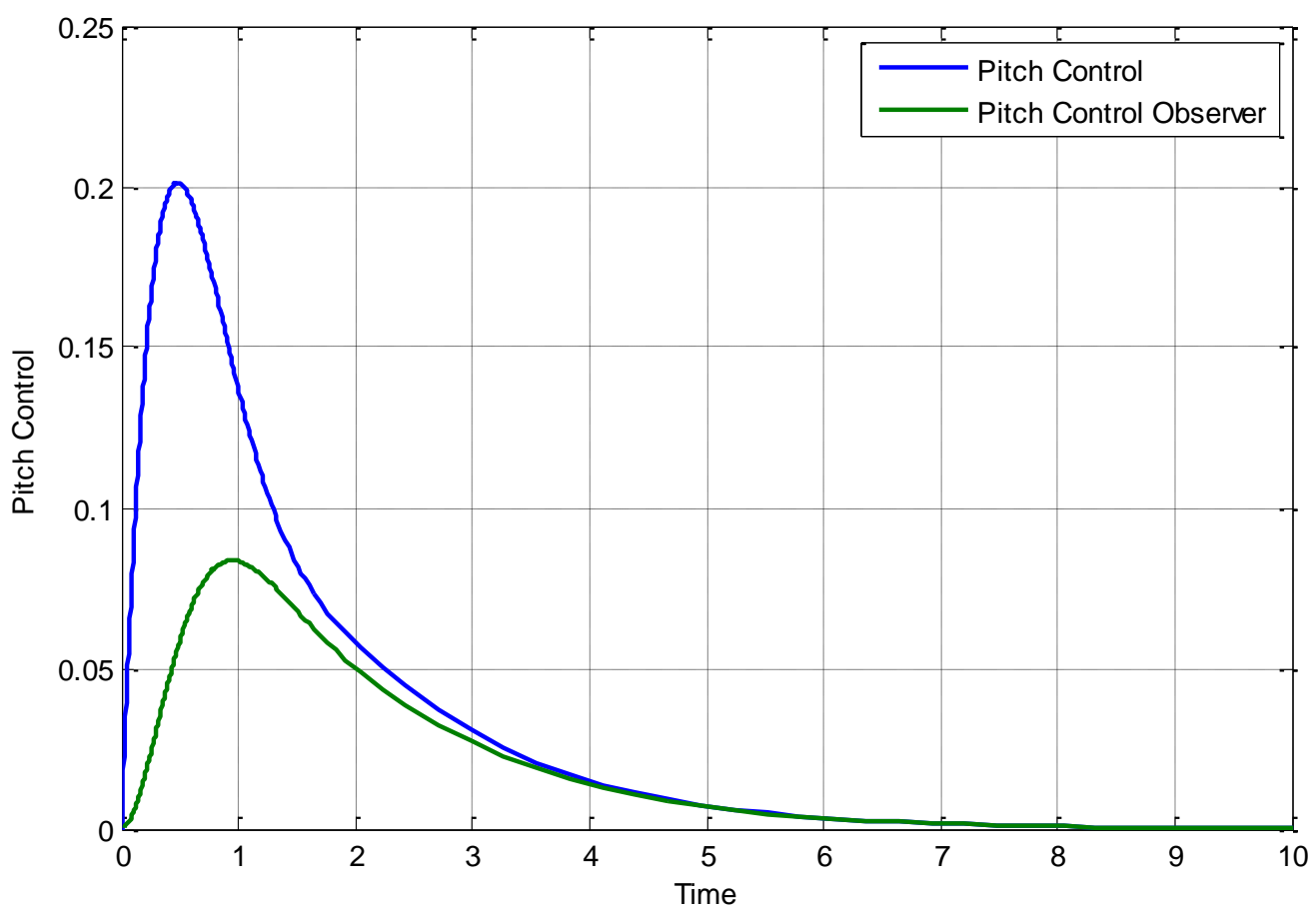

Figure 6. Responses of The Result of Testing the Control Pitch with OFSC Weights $Q=10$ and $R=0.1$

\subsection{Pitch Control Tests Using OSFC with Value of $Q=0.1$ and $R=10$}

The next test was performed by giving a variation of weighting matrix value by decreasing the matrix value of $Q$ and increasing the weighting value of matrix $R$ as shown in the Equation 24 . The response of the third test results is shown in Figure 7.

$$
Q=\left[\begin{array}{ccc}
0.1 & 0 & 0 \\
0 & 0.1 & 0 \\
0 & 0 & 0.1
\end{array}\right] ; R=10
$$

The last test was performed by assigning a decreased value of weight $Q$ and the increased of $R$, the result is that the response of the stabilization process of the pitch angle requires a steady state as much as 48 seconds. Viewed from the time requirement perspective, the test is much longer than the two previous tests. If viewed from the magnitude of the deviation angle, the presence of observer has produced a deviation of 0.04 radians, while without the observer, the produced deviation was 0.21 radians. 
Testing with three variations of weighting on matrices $Q$ and $R$ gives the results of different matrix value of $L$ and $K$. Therefore, it affects the time required for the stabilization process and the magnitude of the deviation angle. The variation values of matrix $Q$ and $R$ affecting the value of the matrix $L$ and $K$ are summarized in Table 1, with matrix $L$ as the observer gain matrix and the matrix $\mathrm{K}$ as the state feedback gain matrix.

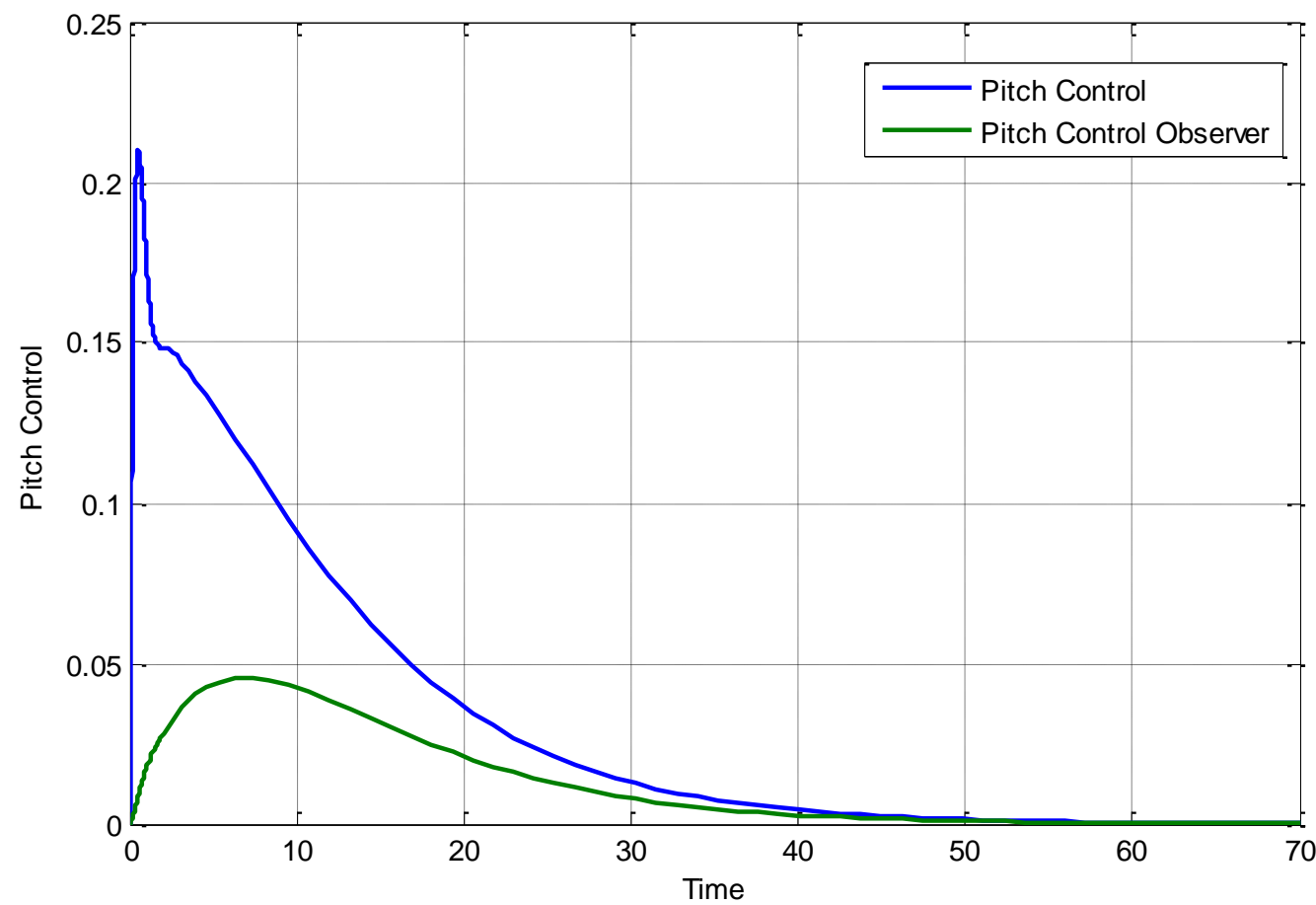

Figure 7. The Response of Pitch Control Test Results with OFSC Weights $Q=0.1$ and $R=10$

Table 1. The Effect of Variations on the Matrix $Q$ and $R$ on the Magnitude Value of the Matrix $L$ and $K$

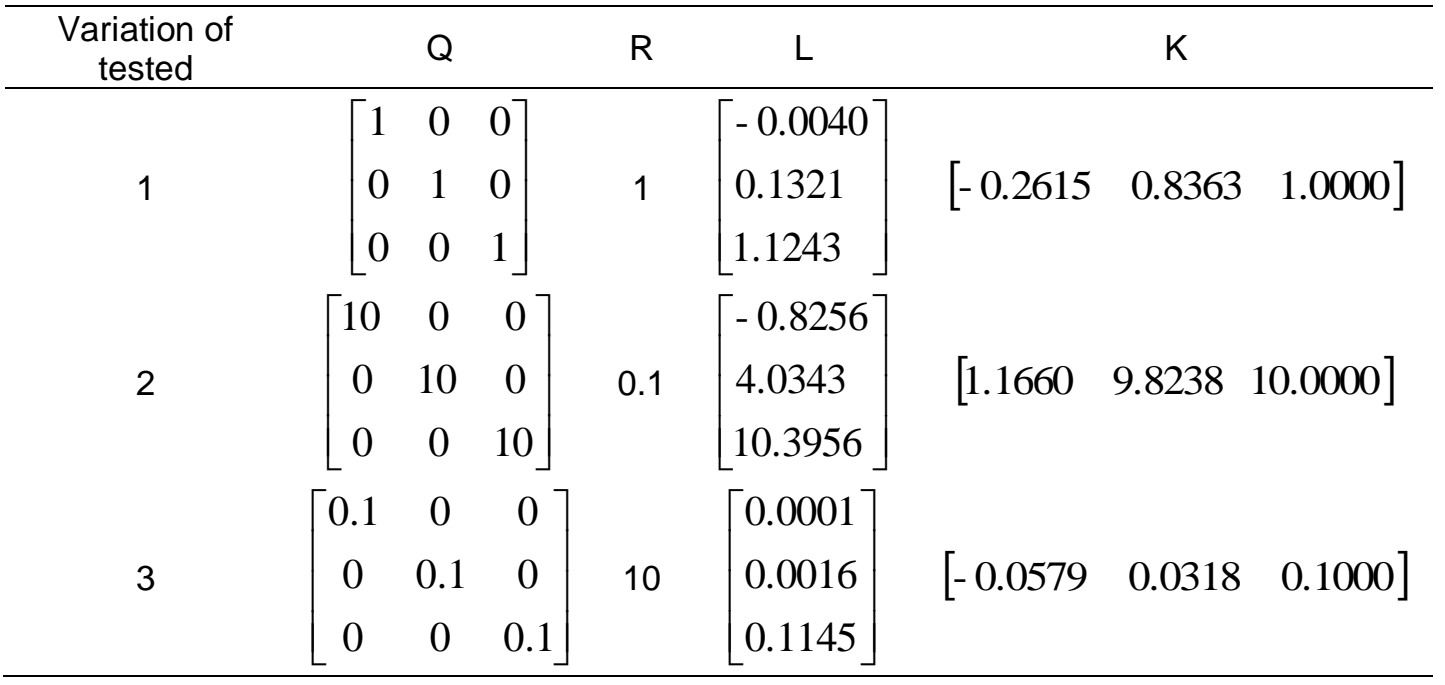

\section{Conclusion}

Based on the test result, it can be concluded that weighted values of $Q$ and $R$ influence the increasing and decreasing value of "the observer gain matrix" (gain L) and "state feedback gain matrix" (gain K). The increment of gain value of $Q$ affects the gain value (gain $K$ ) whereas the gain value of $R$ leads to the decrease in the gain value of $K$. The gain values of $Q$ and $R$ or

KINETIK Vol. 2, No. 4, November 2017: 263-272 
the weighted value must be adjusted to achieve a "steady state" response in a short time duration in minimal angular deviation.

In the case of aircraft pitch control with "observer state feedback control (OSFC)" method employed in this study, the appropriate variation to be used is in the second experiment which has the fastest stabilization processing time of 7 seconds, to reach the condition of "steady state" and the minimum 'pitch' angle deviation value of 0.08 percent.

The use of "observer" has a significant influence on the angular "pitch" angle deviation response. When using "observer," the deviation angle value of "pitch" was 0.08 radians and 0.2 radians without "observer". This finding indicates that "observer" has a significant effect on the "pitch" angle deviation that will directly affect the aircraft stability. The larger the resulting pitch angle deviation will cause the slope of the movement in the x-axis become greater; therefore, an aircraft is prone to fall.

\section{Notation}

$\alpha:$ The Angle of Attack

$q$ :Pitch Rate

$\theta$ : Pitch Angle

$\delta$ : Elevator Deflection

$\rho$ :Density of Air

$S$ :Platform Area of The Wing

$\Omega=\frac{2 U}{c}$

$U$ : Equilibrium Flight Speed

$C_{L}$ : Lift Coefficient

$C_{D}$ : Drag Coefficient

$C_{T}$ : Thrust Coefficient

$C_{M}$ : Moment Coefficient

$C_{W}:$ Weight Coefficient

$\gamma$ : Flight Path Angle

$\sigma=\frac{1}{1+\mu C_{L}}:$ Constant

$i_{y y}$ : Normalized Moment of Inertia

$\eta=\mu \sigma C_{M}:$ Constant

\section{References}

[1] Z. He and W. Xie, "Control of Non-Linear Switched Systems With Average Dwell Time: Interval Observer-Based Framework," IET Control Theory \& Application, Vol. 10, No. 1, Pp. 10-16, Jan. 2016.

[2] A. Tewari, "Advanced Control of Aircraft, Spacecraft and Rockets." Wiley, 2011.

[3] V. V. Klemas, "Coastal and Environmental Remote Sensing from Unmanned Aerial Vehicles: An Overview," Journal of Coastal Research, Vol. 315, No. 5, Pp. 1260-1267, Sep. 2015.

[4] M. Polas and A. Fekih, "A Multi-Gain Sliding Mode Based Controller For The Pitch Angle Control of A Civil Aircraft," 2010 42nd Southeastern Symposium on System Theory (SSST 2010), No. 2, Pp. 96-101, Mar. 2010.

[5] X. Liu, Sheng Sun, Pengjie Lan, and Lifu Du, "Dynamic Surface Attack Angle Control For Aircraft Considering Actuator Position Saturation," in 2016 IEEE Chinese Guidance, Navigation and Control Conference (CGNCC), 2016, Pp. 20-24.

[6] F. T.Johnson, E. T. N, and N. J. Yu, "Thirty years of development and application of CFD at Boeing Commercial Airplanes, Seattle," Computers \& Fluids, Vol. 34, No. 10, Pp. 11151151, Dec. 2005. 
[7] M. V. Cook, "Flight Dynamics Principles: A Linear Systems Approach To Aircraft Stability And Control." Butterworth-Heinemann, 2013.

[8] X.-J. Li and G.-H. Yang, "Dynamic Observer-Based Robust Control and Fault Detection For Linear Systems," IET Control Theory \& Application, Vol. 6, No. 17, Pp. 2657-2666, Nov. 2012.

[9] D. Xue and N. H. El-Farra, "Output Feedback-Based Event-Triggered Control of Distributed Processes with Communication Constraints," in 2016 IEEE 55th Conference on Decision and Control (CDC), 2016, Pp. 4296-4301.

[10] K. Ogata, "Modern Control Engineering," Vol. 17, 2010. 